\title{
Optimization of moth-eye antireflection schemes for silicon solar cells
}

\author{
Stuart A. Boden* and Darren M. Bagnall \\ Nano Group, Electronics and Computer Science, University of Southampton, Highfield, Southampton, SO17 1BJ, UK
}

\begin{abstract}
Nanostructured moth-eye antireflection schemes for silicon solar cells are simulated using rigorous coupled wave analysis and compared to traditional thin film coatings. The design of the moth-eye arrays is optimized for application to a laboratory cell (air-silicon interface) and an encapsulated cell (EVA-silicon interface), and the optimization accounts for the solar spectrum incident on the silicon interface in both cells, and the spectral response of both types of cell. The optimized moth-eye designs are predicted to outperform an optimized double layer thin film coating by approximately $2 \%$ for the laboratory cell and approximately $3 \%$ for the encapsulated cell. The predicted performance of the silicon moth-eye under encapsulation is particularly remarkable as it exhibits losses of only $0.6 \%$ compared to an ideal AR surface. Copyright (C) 2010 John Wiley \& Sons, Ltd.
\end{abstract}

KEYWORDS

Antireflection; Silicon; Moth-eye; Submicron; Surface Texturing; Subwavelength

${ }^{*}$ Correspondence

Stuart A. Boden, Nano Group, Electronics and Computer Science, University of Southampton, Highfield, Southampton, SO17 1BJ, UK. E-mail: sb1@ecs.soton.ac.uk

Received 10 April 2009; Revised 26 October 2009

\section{INTRODUCTION}

Highly absorbing materials are required for solar cells but this often means a correspondingly high refractive index and so a high surface reflectance. Light reflected from the top surface of solar cells can therefore represent a major loss mechanism and so effective reflectance reduction from solar cell surfaces is vital in obtaining high efficiencies. Traditionally, thin film coatings are used for this purpose, with the coating material and thickness chosen to cause destructive interference in reflection for light of a wavelength corresponding to the peak intensity of the solar spectrum [1]. Reflectance is accordingly reduced to very low levels for a narrow range of wavelengths, but effective antireflection (AR) over the broad range of the useful solar spectrum and angles of incidence is not achieved. The situation can be improved using more than one coating, however problems with the availability of suitable materials ultimately limit this approach.

For a more effective antireflective solution, researchers have turned to Nature for inspiration in the form of motheye antireflective schemes; the eyes and wings of certain species of moth are covered in arrays of tapered pillars
(Figure 1a) with a period, $d$ (distance between nearest neighbour pillar centres), and height, $h$, both of about $200 \mathrm{~nm}[2,3]$. A simple understanding of how such arrays can confer an antireflective effect can be gained by considering that the array features are on a scale below the wavelength of incident light and so incoming photons do not react to individual pillars, rather they experience an effective medium whose optical properties are between those of the media on either side of the interface. Tapering of the pillars causes the incident photons to experience a gradual change in refractive index from that of the incident medium to that of the substrate. Reflection only occurs at an abrupt change in the refractive index and so by removing this abrupt change, the moth-eye features confer broadband antireflection (AR) to the interface into which they are formed.

Wilson et al. suggested that the period of a moth-eye array should be as small as possible and the feature height as large as possible to reduce reflection over the widest possible wavelength range [4]. Recently, Huang et al. used a $\mathrm{Ar} / \mathrm{H}_{2}$ plasma etch in an electron cyclotron resonance reactor to form tall, dense conical needle arrays in silicon with needle base widths $<200 \mathrm{~nm}$. The group demonstrated broadband 


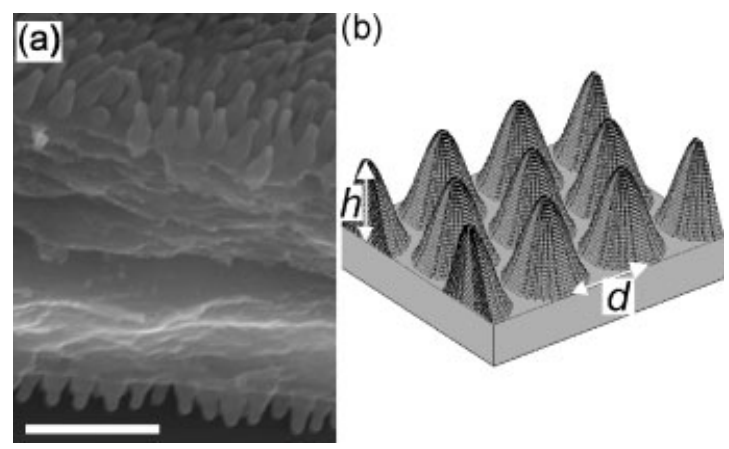

Figure 1. (a) SEM image of the antireflective subwavelength features found on both surfaces of the transparent section of the wing of the Hawkmoth Cryptotympana Aquila (scale bar is $1 \mu \mathrm{m}$ ), (b) Silicon moth-eye array defined in GD-Calc as a stack of 30 strata, each containing cylinders whose radius varies from pillar base to tip.

reflectance of $<3,<0.8$ and $<0.6 \%$ for needle arrays of heights $1.6 \mu \mathrm{m}, 5.5 \mu \mathrm{m}$ and $16 \mu \mathrm{m}$, respectively [5]. However, for PV applications, the resulting textured substrate is required to be suitable for subsequent solar cell fabrication. The formation of a continuous $\mathrm{p}-\mathrm{n}$ junction is hindered by a highly textured front surface and the increase in surface area can lead to increasing surface recombination and so a lowering of the internal quantum efficiency. Furthermore, such tall features are incompatible with thin film devices for which the entire device may only be a few micrometers thick. Therefore, in most examples from the literature, feature height is of the order of several hundred nanometers only.

Researchers have textured quartz [6,7], polymer [8], GaSb [9] and silicon [5,10-13] surfaces with subwavelength moth-eye features and demonstrated very low reflectance across a broad range of wavelengths and angles of incidence. These include Toyota $e t$ al. who fabricated conical shaped features, with a height of $750 \mathrm{~nm}$ and a period of $250 \mathrm{~nm}$ into fused silica using reactive ion etching through a chromium mask defined by electron beam lithography [7]. Reflectance of less than $0.5 \%$ across the wavelength range of $400-800 \mathrm{~nm}$ was achieved. Likewise, Kanamori et al. have demonstrated the texturing of silicon with subwavelength features using dry etching through an e-beam patterned mask[10] and an anodic porous alumina mask $[14,15]$. Reflectance below $2 \%$ for the wavelength range $350-1000 \mathrm{~nm}$ was reported. The group proceeded to fabricate $\mathrm{c}-\mathrm{Si}$ solar cells from substrates textured with $300-400 \mathrm{~nm}$ height features and a period of $100 \mathrm{~nm}$ and in one case achieved an impressive $38 \%$ increase in efficiency (from $10 \cdot 1$ to $13.9 \%$ ) compared to an untextured cell [15]. The group also investigated the angular dependent properties of such texturing and showed that it performs well against more traditional, micron-scale texturing, even at oblique angles of incidence [16].

Subwavelength-scale texturing formed using wet etch techniques, exhibiting the moth-eye effect, has also been successfully applied to PV devices. Koynov et al. used a HF/
$\mathrm{H}_{2} \mathrm{O}_{2} / \mathrm{C}_{2} \mathrm{H}_{5} \mathrm{OH}$ etch, catalysed by $\mathrm{Au}$ nanoclusters on a silicon surface, to form $250 \mathrm{~nm}$ high features in c-Si, mc-Si and a-Si substrates [17]. Reflectance was reduced to $<5 \%$ for wavelengths from $350-1000 \mathrm{~nm}$ for the crystalline silicon substrates. Subsequent cell fabrication on mc-Si substrates resulted in a $36-42 \%$ increase in photocurrent compared to untextured cells [18]. In this case, the more random nature of the etch technique resulted in a range of lateral spacings from 50 to $100 \mathrm{~nm}$. Using a similar technique, Nishioka et al. fabricated subwavelength structures in silicon with a range of heights and average periods by varying the etch time [19]. For a height of $200 \mathrm{~nm}$, the average reflectance increased from 4 to $9 \%$ as the period increased from 100 to $170 \mathrm{~nm}$. These studies all suggest that bio-inspired subwavelength texturing can indeed reduce surface reflectance of PV materials over a broad range of wavelengths and angles of incidence and that it can be successfully applied to enhance the performance of solar cells. The question now arises as to whether it is possible to tune the parameters of such textures for optimum performance of a particular solar cell under standard solar conditions.

Previously, we presented results from rigorous coupled wave analysis (RCWA) simulations showing that the motheye array pillar shape, height and period all affect the reflectance properties of the surface [13]. Furthermore, we suggested that these parameters could be tuned to optimize the antireflective properties of a silicon moth-eye surface for a specific incident spectrum. Sai et al. have also used RCWA to study the effect of changing the ratio of the height to the period (aspect ratio) on the average reflectance of a silicon surface, weighted by the standard solar spectrum [20]. They report a decrease in weighted reflectance with increasing period from 100 to $400 \mathrm{~nm}$ for a fixed aspect ratio. In this work, we decouple the effects of changing period and the height and describe a theoretical optimization of the period of moth-eye arrays for silicon solar cell AR applications. The resulting predicted performances are compared to those of optimized thin film coatings. The analysis is done for a high efficiency c-Si laboratory cell (air-silicon interface) and typical screen-printed, encapsulated cell (EVA-silicon interface).

\section{CAlCulating REFLeCtAnCe}

Moth-eye arrays in silicon are modelled using RCWA implemented in the commercial software package, GDCalc [21]. The hexagonally arranged pillars that comprise the moth-eye arrays are defined as stacks of cylinders with varying radii to represent the tapering of the structures. To reduce the number of variables, the pillar profile was fixed, using a cosine-based function to describe the variation of the pillar radius from base to tip, which results in the biomimetic shape shown in Figure 1b. The reflected orders are summed to give the total reflectance from the interface.

To validate the use of RCWA with this type of surface, a silicon moth-eye sample fabricated using electron beam lithography and reactive ion etching (for fabrication details, 
see $[12,13])$ was modelled in GD-Calc. The period and pillar profile were determined from an SEM image of the fabricated structure (Figure 2a) and these parameters were used to reproduce the structure in the modelling software (Figure 2b). Reflectance measurements were obtained from the fabricated sample using a reflectance probe technique and these were compared to the calculated reflectance spectrum. The structure is not optimized and so exhibits only a modest AR effect, however, Figure 2c clearly shows there is an excellent match between the measured and calculated reflectance spectra, which suggests we can confidently use the RCWA approach to accurately predict the reflectance properties of silicon moth-eye arrays.

\section{SPECTRAL DATA}

Spectral data were obtained from an implementation of the Bird Simple Spectral Model, SPCTRAL2, available from the National Renewable Energy Laboratory (NREL) [22]. This program allows longitude, latitude and slope to be specified along with the date and time (other inputs are listed in Table I). Details on the manipulation of this data to obtain photon flux density (PFD) for a range of wavelengths and angles of incidence, which represents the incident solar spectra over half a day, are provided in Reference [23]. RCWA calculations are computationally

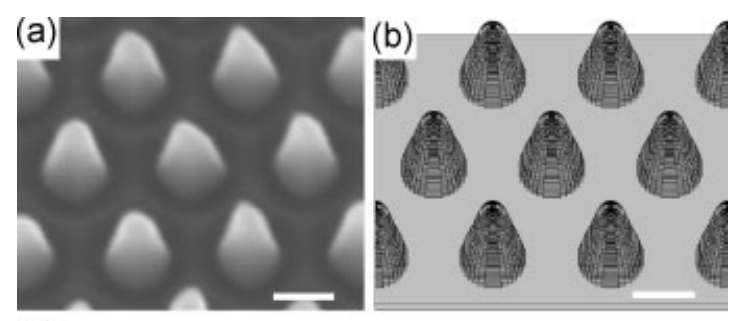

(c)

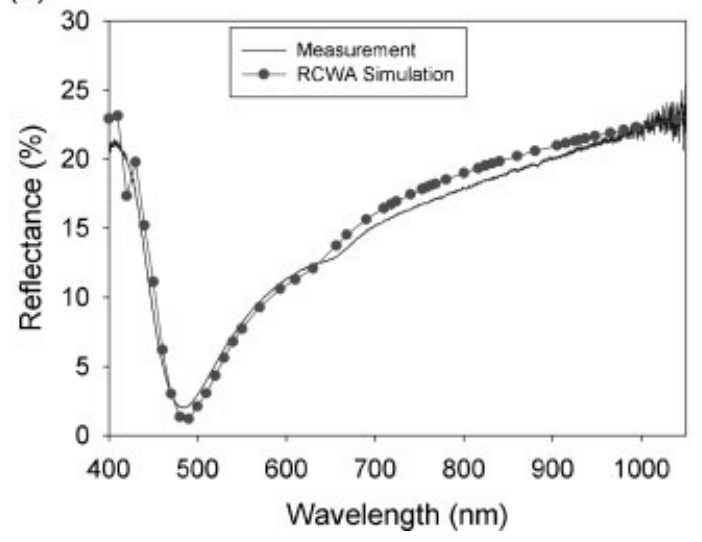

Figure 2. (a) SEM image of silicon moth-eye sample with period of approximately $191 \mathrm{~nm}$ and a height of approximately $189 \mathrm{~nm}$, tilted at an angle of $28^{\circ}$, (b) corresponding moth-eye defined in RCWA simulation software, (c) simulated and measured reflectance spectra for this silicon moth eye. Scale bars are $100 \mathrm{~nm}$.
Table I. Input parameters used in SPCTRAL2 program [22].

\begin{tabular}{lc}
\hline Latitude $\left(^{\circ}\right)$ & 0 \\
Longitude $\left(^{\circ}\right)$ & 0 \\
Aerosol optical depth & $0 \cdot 27$ \\
Alpha & $1 \cdot 14$ \\
Albedo (surface reflectance) & $0 \cdot 2$ \\
Total column ozone (cm) & $0 \cdot 34$ \\
Total precipitable water Vapour $(\mathrm{cm})$ & $1 \cdot 42$ \\
Slope $\left(^{\circ}\right.$ ) & 0 \\
Surface pressure $(\mathrm{mB})$ & 1013.25 \\
Day of the year & 79 (Mar 20) \\
\hline
\end{tabular}

more demanding than the transfer matrix approach used previously, so for this optimization, the spectrum from the time of day when the solar irradiance is most intense is used (normal incidence, around midday, Figure 3). In the case of the encapsulated cell, the incident spectrum is altered by the presence of the glass and EVA layers. This is accounted for by including reflectance from the glass surface and absorption in the EVA in the approach described previously [23], which results in the modified spectrum shown in Figure 3.

\section{INTERNAL QUANTUM EFFICIENCY}

Internal quantum efficiency data for a high efficiency laboratory silicon solar cell were obtained from Wang and coworkers who modelled a passivated emitter rear locallydiffused (PERL) solar cell [24]. Well passivated front and rear surfaces ensure that this cell exhibits a very high IQE across the whole spectral range. For the simulations involving an encapsulated cell, the IQE curve for a typical screen-printed monocrystalline solar cell, obtained from Ebong et al., was used [25]. This was corrected to remove absorption in the thin-film coating applied to this cell [23]. The resulting IQE data are shown in Figure 4.

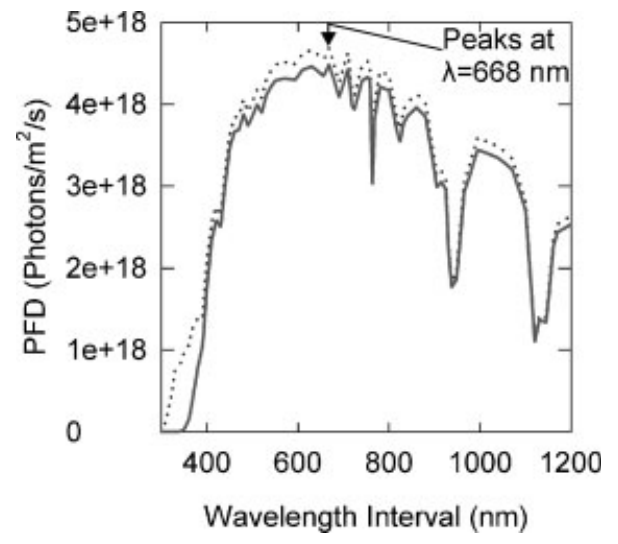

Figure 3. Total normal incidence PFD incident at the silicon interface for lab cell (dotted line) and encapsulated cell (solid line). 


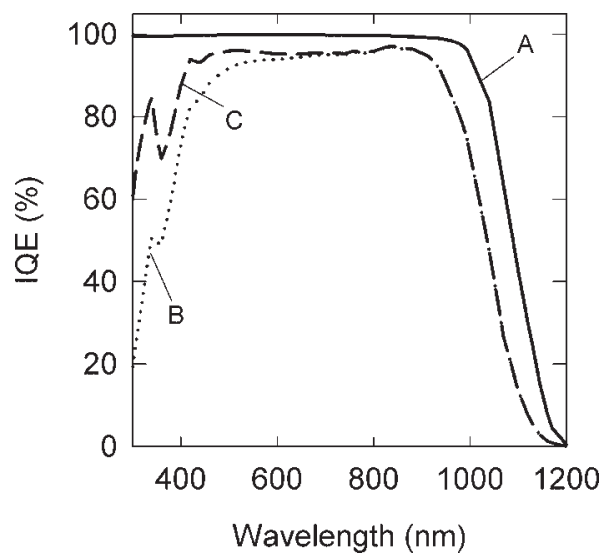

Figure 4. Internal quantum efficiency curves for silicon solar cells: A. High efficiency PERL cell (used in laboratory cell simulations) [24], B. screen printed cell [25], C. screen printed cell with effect of absorption in AR coating removed (used in encapsulated cell simulations).

\section{MOTH-EYE ARRAY OPTIMIZATION}

Reflectance is calculated for a range of periods and pillar heights for a fixed wavelength, chosen as the wavelength at which the peak in the PFD occurs, which is, for both cells, $668 \mathrm{~nm}$ (see Figure 3). Contour plots of reflectance at this wavelength for air-silicon and EVAsilicon interfaces as period and pillar height are varied as shown in Figure 5.

The results show that for both cells, if the period is decreased below $200 \mathrm{~nm}$, the reflectance increases rapidly for all pillar heights, with the effect being greater at smaller pillar heights. This is in agreement with Sai et al. who found that for periods less than $200 \mathrm{~nm}$, much higher aspect ratios are required to achieve low reflectance [20]. The reflectance increases for larger periods too as the features move out of the subwavelength regime for the shorter wavelengths and diffraction orders begin to emerge. This effect is also in agreement with the work of Sai et al. [20].

It is clear that a valley in reflectance exists in which low reflectance $(<1 \%)$ can be achieved for modest pillar heights. This represents a sweet spot for the design of moth-eye AR surfaces because the larger the pillar height, the more difficult (and costly) fabrication of the moth-eye array becomes. Cell fabrication processes such as junction diffusion are also more challenging in surfaces textured with taller pillars. In addition, larger pillar heights mean a larger surface area of silicon and so a possible increase in surface recombination which could negate any benefits conferred by the reduction in reflectance.

A pillar height of $400 \mathrm{~nm}$ (marked by the dashed lines on the contour plots on Figure 5) was chosen as a compromise between low reflectance and fabrication and surface area considerations. Plots showing cross-sections through Figure 5 at a height of $400 \mathrm{~nm}$ are presented in Figure 6 . The reflectance lies well below $1 \%$ for a pillar height of $400 \mathrm{~nm}$ in the low reflectance region centred on a period of approximately $250 \mathrm{~nm}$ for the air-silicon interface and approximately $290 \mathrm{~nm}$ for the EVAsilicon interface. The simplest method for determining optimum periods for moth-eyes on the laboratory and encapsulated cells would be to choose the periods corresponding to the minima in Figure 6, but this would mean optimizing only for the peak wavelength in the solar spectrum. A truer optimum period can be determined by optimizing for the entire solar spectrum when the solar irradiance experienced by a cell is at its most intense (i.e. when direct sunlight is incident normal to the surface so $\mathrm{AOI}=0$ ). This can be done by calculating reflectance spectra at an AOI of $0^{\circ}$ for a range of periods. By combining these with IQE data (Figure 4) and the normal incidence PFD (Figure 3), the (a)

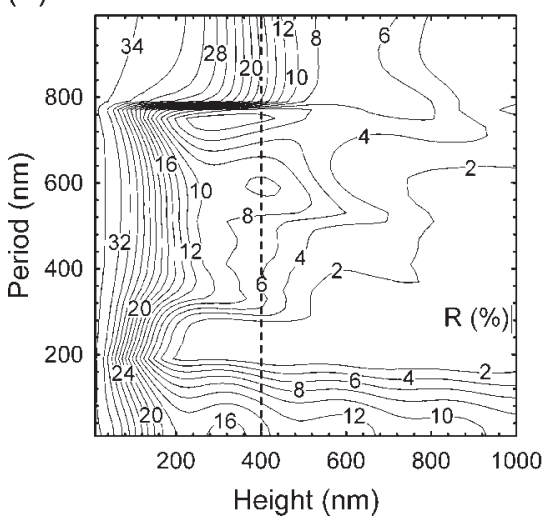

(b)

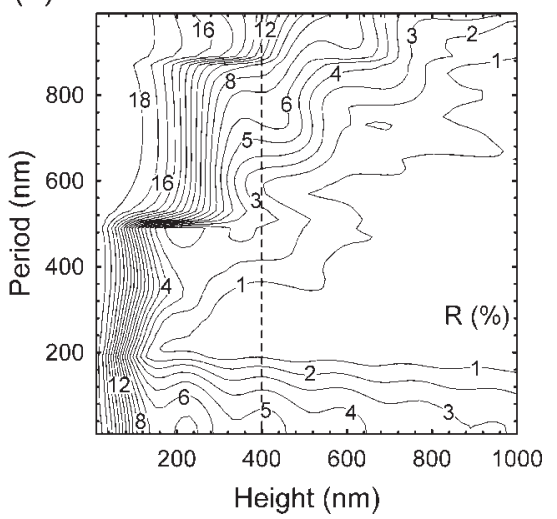

Figure 5. Parameter map showing the simulated variation of reflectance with period and pillar height for a silicon moth-eye array at a wavelength of $668 \mathrm{~nm}$, which corresponds to the peak in PFD spectrum (Figure 3): (a) air-silicon interface, (b) EVA-silicon interface. The dashed lines show the cross-sections taken when plotting the graph in Figure 6. 
(a)

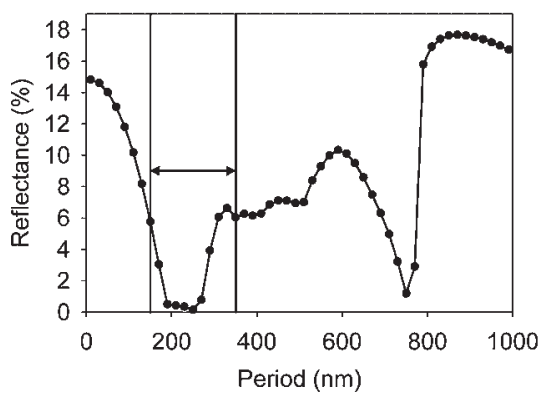

(b)

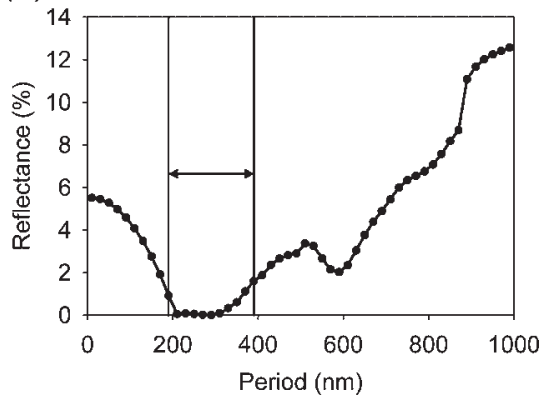

Figure 6. Cross-sections through the contour plots in Figure 5 at a height of $400 \mathrm{~nm}$, showing reflectance as a function of the period of a silicon moth-eye array, for a wavelength of $668 \mathrm{~nm}$ : (a) laboratory cell, (b) encapsulated cell. The period ranges over which the optimizations were carried out are shown by the double-ended arrows.

maximum short- circuit current, $J_{s c}$, can be calculated and used as a figure of merit for the optimization:

$J_{s c}=q \int_{\lambda=300 \mathrm{~nm}}^{\lambda=1240 \mathrm{~nm}}[\operatorname{PFD}(\lambda)(1-R(\lambda)) \operatorname{IQE}(\lambda)] \mathrm{d} \lambda$

The optimum periods for each type of interface will be those resulting in the highest values of $J_{s c}$. Reflectance spectra for the period ranges identified above are presented in Figure 7 and the variations in $J_{s c}$ with period calculated using these reflectance spectra are shown in Figure 8. In this analysis, we find that the optimum period for the laboratory cell is $280 \mathrm{~nm}$ and the optimum period for the encapsulated cell is $312 \mathrm{~nm}$.

\section{COMPARISON WITH THIN FILIM AR COATINGS}

Previously we reported on the theoretical optimization of both single and double layer thin film coatings, for optimum AR over the range of wavelengths and angles of incidence experienced by a typical solar cell from sunrise
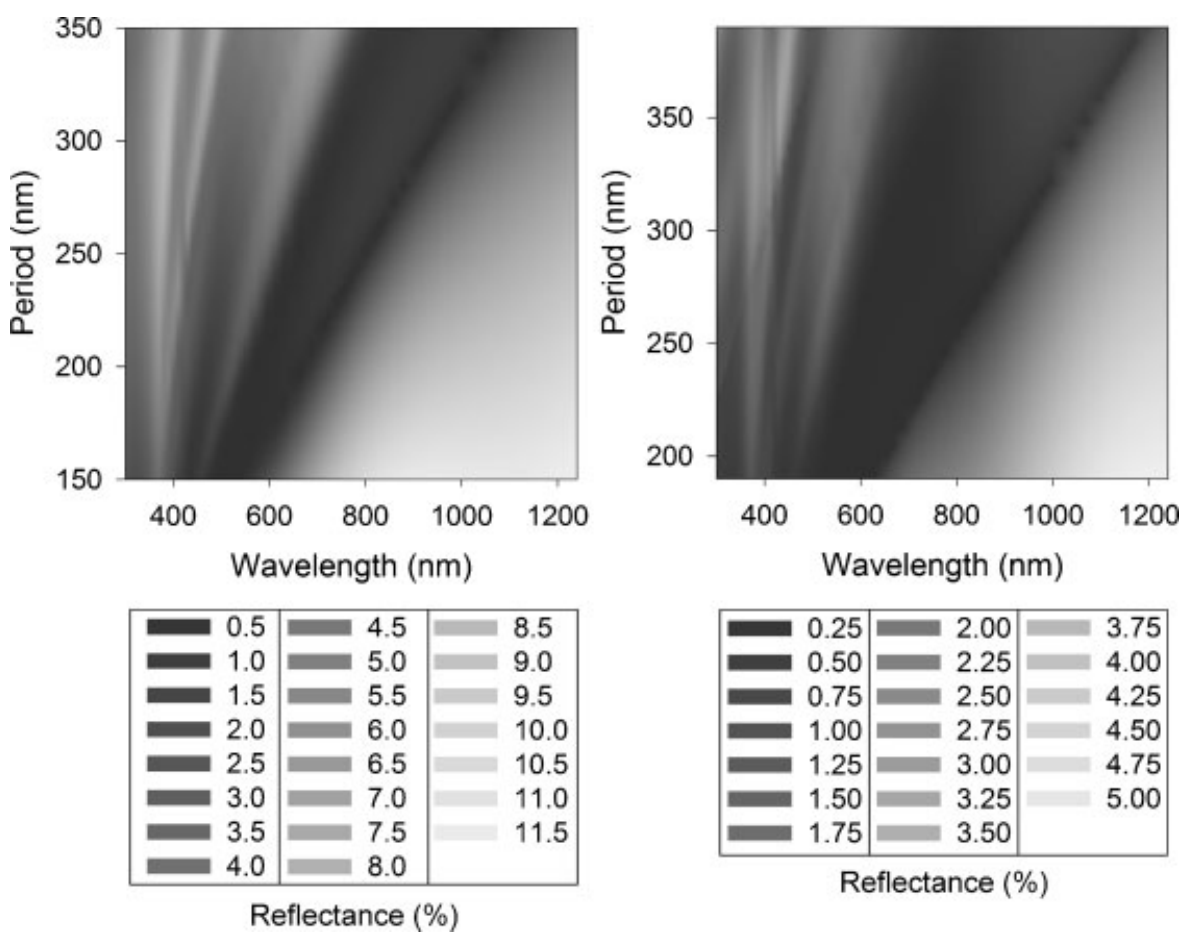

Figure 7. Contour plot of simulated reflectance spectra as the period of the moth-eye array is varied in $2 \mathrm{~nm}$ steps over the low reflectance ranges identified in Figure 6. The height is fixed at $400 \mathrm{~nm}$. (a) air-silicon interface, (b) EVA-silicon interface. 

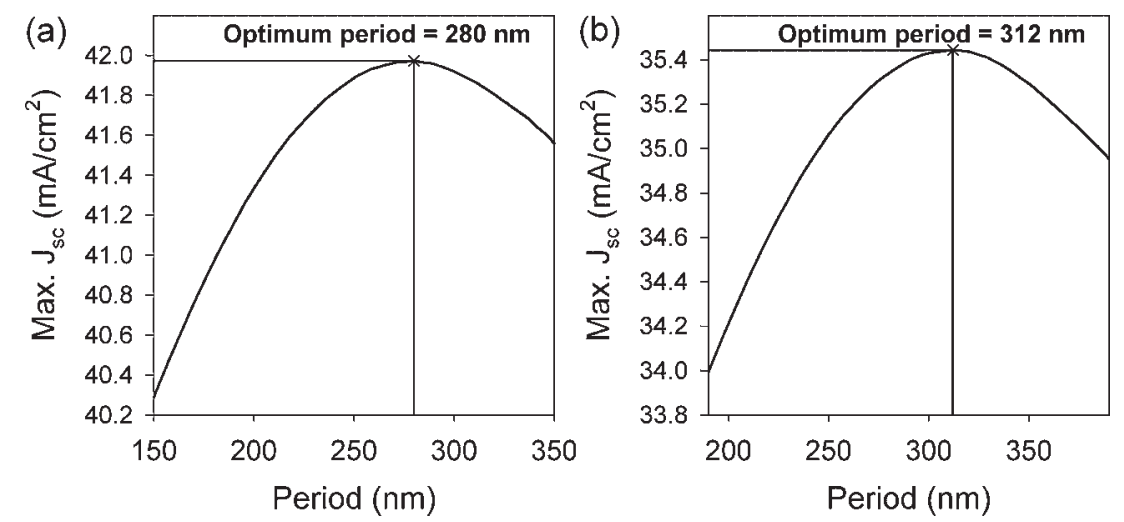

Figure 8. Calculated short-circuit current produced by a cell at peak solar intensity as a function of period, with the optimum periods for each cell type shown, (a) laboratory cell, (b) encapsulated cell.

to sunset [23]. For a direct comparison with these optimized thin film coatings, transmittance spectra for a range of AOIs are calculated for moth-eye arrays with optimum periods. This data is then combined with sunrise to sunset spectral data $(\operatorname{PFD}(\lambda, \theta))$ and the IQE data shown in Figure 4 to calculate the average short-circuit current, $J_{\text {scAve }}$, following the procedure described in
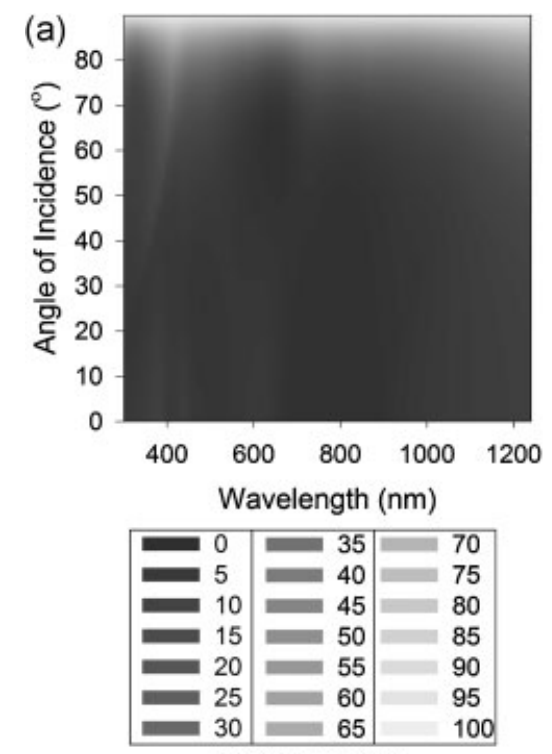

Reflectance (\%)

(c)

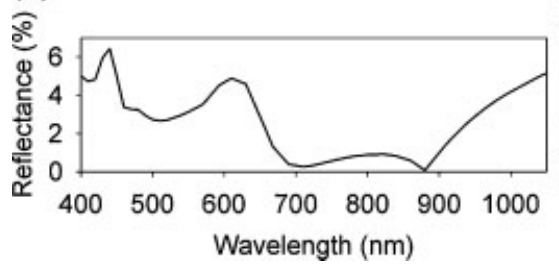

Reference [23]. This was carried out for both the laboratory and encapsulated cells, positioned on a horizontal surface at the equator; the resulting values of $J_{\text {scave }}$ can be used to compare these different types of AR scheme.

Reflectance variations with wavelength and incident angle for both types of cell are presented in Figure 9. Reflectance of the textured interfaces remains low up to

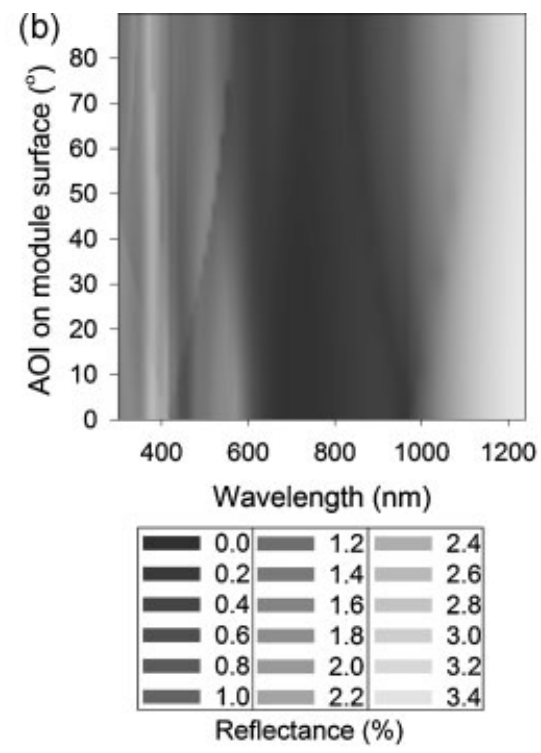

(d)

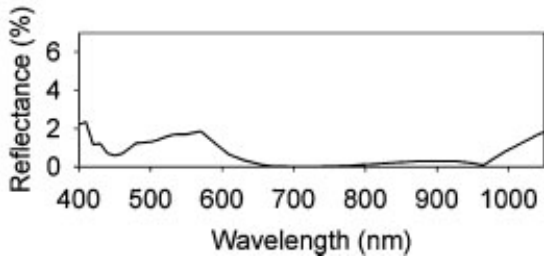

Figure 9. Calculated reflectance versus wavelength and angle of incidence for optimized moth-eye designs, (a) air-silicon interface (period $=280 \mathrm{~nm}$ ), (b) EVA-silicon interface (period $=312 \mathrm{~nm}$ ). (c) and (d) give the reflectance spectra at normal incidence for the airsilicon and EVA-silicon interfaces, respectively. 
high angles of incidence, in agreement with Sai et al. [16], which is important for PV applications for which the incidence angle of direct sunlight varies considerably throughout a day. Values for $J_{s c A v e}$ of the optimized motheye surface on the laboratory cell are presented in Table II. For comparison, $J_{\text {scAve }}$ values for a $\mathrm{Si}_{3} \mathrm{~N}_{4}$ single layer coating (SLAR) and $\mathrm{SiO}_{2} / \mathrm{TiO}_{2}$ double layer coating (DLAR), with optimal thicknesses [23] are included, along with the value for an ideal AR surface (i.e. zero reflectance for all angles and wavelengths). Percentage losses compared with an ideal AR surface are also included. Values for the encapsulated cell are given in Table III compared in this case to an optimum thickness $\mathrm{TiO}_{2}$ SLAR and $\mathrm{SiN}_{\mathrm{X}} / \mathrm{TiO}_{2}$ DLAR. A graphical illustration of these values is shown in Figure 10. The calculations show that moth-eye arrays can outperform traditional thin film coatings: The optimized moth-eye designs are predicted to outperform the best DLARs by $2 \%$ and $3 \%$ for the laboratory and encapsulated cells, respectively. The inclusion of a moth-eye texture to the EVA-Si interface in an encapsulated cell is shown to be particularly effective as it is predicted to exhibit losses of only $0.6 \%$ compared to an ideal AR surface.

\section{DISCUSSION}

The calculations presented here demonstrate that subwavelength texturing can outperform optimized thin film AR coatings in solar cell applications. A comparison with published data reveals that even better performances than those predicted can be achieved. The measured reflectance spectrum shows that the AR surface reported by Sai et al. exhibits a reflectance less than $2 \%$ across the wavelength range of 350-1000 nm [15]. Likewise, Koynov et al. and Nishioka et al. report textured surfaces with reflectance less than 5\% across the wavelength range of $350-1000 \mathrm{~nm}$, with feature heights of only $200-250 \mathrm{~nm}$ $[17,19]$. In contrast, the calculated reflectance spectrum of our $400 \mathrm{~nm}$ high array (Figure 9c) is only $<2 \%$ between wavelengths of 660 and $920 \mathrm{~nm}$. We suggest two explanations for this:

(i) The complexity of the RCWA calculations has limited the number of parameters that can be optimized in a reasonable time. In particular, the profile of the pillars

Table II. Performance of laboratory cell (air-silicon interface) employing various AR schemes. $J_{\text {scAve }}$ is the predicted average short-circuit current produced by the cell over a day. '\% loss' is the reduction in $J_{\text {scave }}$ compared to an ideal AR coating $(100 \%$ transmission for all wavelengths and AOIs).

\begin{tabular}{lcr}
\hline AR Scheme & $J_{\text {scAve }}\left(\mathrm{mA} / \mathrm{cm}^{2}\right)$ & $\%$ Loss \\
\hline Ideal AR coating & 27.66 & 0 \\
$\mathrm{Si}_{3} \mathrm{~N}_{4} \mathrm{SLAR}$ & 23.82 & 13.9 \\
$\mathrm{SiO}_{2} / \mathrm{TiO}_{2}$ DLAR & 25.65 & 7.3 \\
Optimized Moth-eye $($ period $=280 \mathrm{~nm})$ & 26.18 & 5.3 \\
\hline
\end{tabular}

Table III. Performance of encapsulated cell (EVA-silicon interface) employing various AR schemes. $J_{\text {scAve }}$ is the predicted average short-circuit current produced by the cell over a day. ' $\%$ loss' is the reduction in $J_{\text {scAve }}$ compared to an ideal AR coating (100\% transmission for all wavelengths and AOIs).

\begin{tabular}{lcr}
\hline AR Scheme & $J_{\text {scAve }}\left(\mathrm{mA} / \mathrm{cm}^{2}\right)$ & $\%$ Loss \\
\hline Ideal AR coating & 22.36 & 0 \\
$\mathrm{TiO}_{2} \mathrm{SLAR}$ & 21.34 & 4.6 \\
$\mathrm{SiN}_{\times} / \mathrm{TiO}_{2}$ DLAR & 21.59 & 3.5 \\
Optimized moth-eye $($ period $=312 \mathrm{~nm})$ & 22.22 & 0.62 \\
\hline
\end{tabular}

in this study was rather arbitrarily chosen as one that resembles a natural moth-eye surface. It seems that the experimentally realised pillar profiles from the literature perform better than the biomimetic profile chosen for this study. This highlights the importance of being inspired by but not limited to designs from Nature.

(ii) The more random nature of the experimental surfaces may lead to a better overall performance than a perfectly regular array from simulations. For the fabricated structures, periodicity is a range rather than a discreet value and this could result in the extension of the region of low reflectance across a broader wavelength range.

The optimizations presented here can be used to guide experimental design choices, with the added advantage that the natural variations conferred by the fabrication process will lead to even better performances than that predicted by simulation.

Passivation of moth-eye surfaces has not been considered in this study because of the difficulty of including a passivating layer in the GD-Calc simulations. The motheye surfaces will need to be well passivated to minimize the

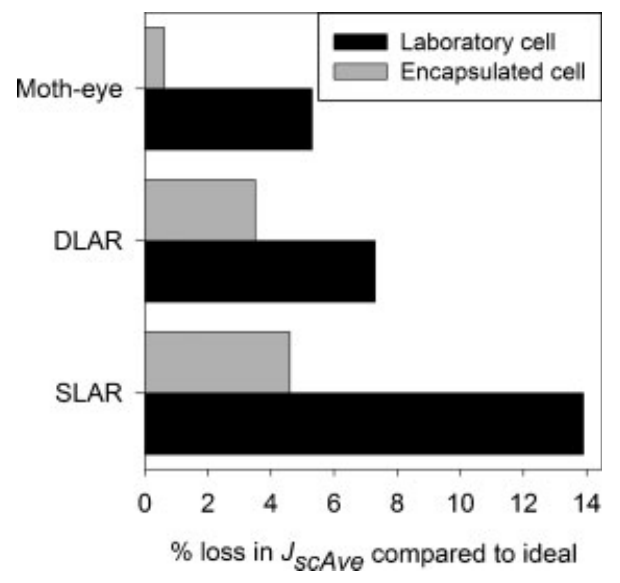

Figure 10. Comparison of optimized moth-eye surfaces with the best thin-film AR coatings in terms of percentage loss in average short-circuit current produced by the laboratory and encapsulated cell, compared to these cells with an ideal AR coating. Values are given in Table II and Table III. 
detrimental effect of increased surface recombination as a result of increasing the surface area and introducing damage during the etch processes. Indeed, Sai et al. have demonstrated that the IQE of subwavelength textured cells is substantially degraded at shorter wavelengths if the surface is left unpassivated [16]. Nevertheless, our previous study predicted that the addition of a thin passivating layer would have only a minimal effect on the properties of thinfilm AR coatings [23] and so is unlikely to significantly alter the reflectance of moth-eye arrays. However, the concern remains as to whether or not moth-eye surfaces can be adequately passivated to ensure minimal surface recombination losses.

Linked to the previous point is the question of the optimum pillar height, which for this study was chosen at $400 \mathrm{~nm}$. Increasing the height will lead to a further reduction of reflectance, but at the expense of subsequent cell fabrication ease and with the danger of further enhancing surface recombination. This remains a topic for further study.

Another widely implemented AR method is to texture on the scale above the wavelength of light to increase coupling into the substrate by forcing incident light to undergo multiple reflections. An example of this is the anisotropically etched pyramidal texturing scheme used on many solar cells [26,27]. A detailed and systematic comparison of moth-eye arrays, deep textured surfaces and textured surfaces coated with thin film AR layers is yet to be completed, though a brief comparison of our data with the reflectance data provided in Reference [28] seems to indicate similar reflectance performance. It is perhaps unlikely that moth-eye schemes will replace the rather convenient inverted pyramid texturing scheme for tradition silicon wafer solar cells, however, texturing with larger features is often incompatible with thin-film devices, which suggests that moth-eye arrays could provide effective AR coatings for second generation solar cells.

This work is focused on optimizing for surface reflection reduction of light most useful to a solar cell. Designs that are in the subwavelength regime for the entire solar spectrum have been shown to be the most effective in this respect. There may be other optimum designs when taking into consideration absorption of light and device thickness. Here, light trapping effects such as scattering or diffraction conferred by larger scale features could be considered [20].

The main barrier to the large scale implementation of moth-eye arrays as effective AR schemes for commercial cells is the cost of texturing on such a small scale. Electron beam lithography, whilst effective in the precise definition of arrays of nanoscale features, is prohibitively expensive for patterning areas required for solar cells. Nevertheless, rapidly developing techniques such as nanoimprint lithography (NIL) [29,30], nanosphere lithography [31,32] and even cheaper, maskless etch techniques [17,19] could pave the way for cheap artificial moth-eye arrays over large areas and so provide a commercially feasible as well as a technically effective AR scheme for all types of solar cell.

\section{CONCLUSION}

In summary, a technique based on RCWA has been used to theoretically optimize the period of moth-eye arrays for antireflective surfaces on laboratory and encapsulated silicon solar cells. The optimized designs are predicted to outperform the best DLAR coatings by approximately $2 \%$ for the laboratory cell and approximately $3 \%$ for the encapsulated cell, demonstrating great promise as alternatives to standard AR schemes for photovoltaics. Application to an encapsulated cell is predicted to be particularly effective, with only $0.6 \%$ less average shortcircuit current produced over a day compared to an ideal AR surface. Comparisons to experimentally realised subwavelength textures from the literature suggest that even better AR performances are possible with different pillar profiles and less regular feature spacings.

\section{REFERENCES}

1. Zhao J, Green MA. Optimized antireflection coatings for high-efficiency silicon solar-cells. IEEE Transactions on Electron Devices 1991; 38(8): 19251934.

2. Bernhard CG. Structural and functional adaption in a visual system. Endeavour 1967; 26: 79-84.

3. Vukusic P, Sambles JR. Photonic structures in biology. Nature 2003; 424(6950): 852-855.

4. Wilson SJ, Hutley MC. The optical properties of 'moth eye' antireflection surfaces. Opt. Acta 1982; 29(7): 993-1009.

5. Huang YF, Chattopadhyay S, Jen Y-J, Peng C-Y, Liu T-A, Hsu Y-K, Pan C-L, Lo H-C, Hsu C-H, Chang Y-H, Lee C-S, Chen K-H, Chen L-C. Improved broadband and quasi-omnidirectional anti-reflection properties with biomimetic silicon nanostructures. Nature Nanotechnology 2007; 2(12): 770-774.

6. Enger RC, Case SK. Optical-elements with ultrahigh spatial-frequency surface corrugations. Applied Optics 1983; 22(20): 3220-3228.

7. Toyota H, Takahara K, Okano M, Yotsuya T, Kikuta H. Fabrication of microcone array for antireflection structured surface using metal dotted pattern. Japanese Journal of Applied Physics Part 2 2001; 40(7B): L747-L749.

8. Clapham PB, Hutley MC. Reduction of lens reflection by moth eye principle. Nature $1973 ; 244(5414)$ : 281282.

9. Min WL, Betancourt AP, Jiang P, Jiang B. Bioinspired broadband antireflection coatings on GaSb. Applied Physics Letters 2008; 92(14): 141109.

10. Kanamori Y, Sasaki M, Hane K. Broadband antireflection gratings fabricated upon silicon substrates. Optics Letters 1999; 24(20): 1422-1424.

11. Lalanne P, Morris GM. Antireflection behavior of silicon subwavelength periodic structures for visible light. Nanotechnology 1997; 8(2): 53-56. 
12. Boden SA, Bagnall DM. Bio-mimetic nanostructured surfaces for near-zero reflection sunrise to sunset, PVSAT-3, Durham, UK, 2007.

13. Boden SA, Bagnall DM. Tunable reflection minima of nanostructured antireflective surfaces. Applied Physics Letters 2008; 93: 133108.

14. Kanamori Y, Hane K, Sai H, Yugami H. $100 \mathrm{~nm}$ period silicon antireflection structures fabricated using porous alumina membrane mask. Applied Physics Letters 2001; 78(2): 142-143.

15. Sai H, Fujii H, Kanamori Y, Arafune K, Ohshita Y, Yugami H, Yamaguchi M. Numerical analysis and demonstration of submicron antireflective textures for crystalline silicon solar cells. Proceedings of the IEEE 4th World Conference on Photovoltaic Energy Conversion 2006; Hawaii, 1191-1194.

16. Sai H, Fujii H, Arafune K, Ohshita Y, Kanamori Y, Yugami H, Yamaguchi M. Wide-angle antireflection effect of subwavelength structures for solar cells. Japanese Journal of Applied Physics 2007; 46(6A): 3333-3336.

17. Koynov S, Brandt MS, Stutzmann M. Black nonreflecting silicon surfaces for solar cells. Applied Physics Letters 2006; 88: 203107.

18. Koynov S, Brandt MS, Stutzmann M. Black multicrystalline silicon solar cells. Physica Status Solidi (Rapid Research Letters) 2007; 1(2): R53-R55.

19. Nishioka K, Horita S, Ohdaira K, Matsumura H. Antireflection subwavelength structure of silicon surface formed by wet process using catalysis of single nano-sized gold particle. Solar Energy Materials \& Solar Cells 2008; 92: 919-922.

20. Sai H, Kanamori Y, Arafune K, Ohshita Y, Yamaguchi M. Light trapping effect of submicron surface textures in crystalline $\mathrm{Si}$ solar cells. Progress in Photovoltaics: Research and Applications 2007; 15: 415-423.

21. Johnson KC. GD-Calc. 2005; http://software.kjinnovation.com/GD-Calc.html.

22. Bird RE, Riordan CJ. Simple solar spectral model for direct and diffuse irradiance on horizontal and tilted planes at the earth's surface for cloudless atmospheres. Journal of Climate and Applied Meteorology
1986; 25(1): 87-97. (http://rredc.nrel.gov/solar/models/ spectral/).

23. Boden SA, Bagnall DM. Sunrise to sunset optimization of thin film antireflective coatings for silicon solar cells. Progress in Photovoltaics: Research and Applications 2009; 17: 241-252.

24. Wang A, Zhao J, Green MA. 24\% efficient silicon solar cells. Applied Physics Letters 1990; 57(6): 602604.

25. Ebong A, Hilali M, Rohatgi A, Meier D, Ruby DS. Belt furnace gettering and passivation for n-web silicon for high-efficiency screen-printed frontsurface-field solar cells. Progress in Photovoltaics: Research and Applications 2001; 9: 327-332.

26. Papet P, Nichiporuk O, Kaminski A, Rozier Y, Kraiem J, Lelievre J-F, Chaumartin A, Fave A, Lemiti M. Pyramidal texturing of silicon solar cell with TMAH chemical anisotropic etching. Solar Energy Materials and Solar Cells 2006; 90(15): 2319-2328.

27. Zhao JH, Wang A, Green MA. $19.8 \%$ efficient "honeycomb" textured multicrystalline and $24.4 \%$ monocrystalline silicon solar cells. Applied Physics Letters 1998; 73(14): 1991-1993.

28. Parretta A, Sarno A, Tortora P, Yakubu H, Maddalena P, Zhao J, Wang A. Angle-dependent reflectance measurements on photovoltaic materials and solar cells. Optics Communications 1999; 172(1-6): 139151.

29. Ting C-J, Chang F-Y, Chen C-F, Chou CP. Fabrication of an antireflective polymer optical film with subwavelength structures using a roll-to-roll microreplication process. Journal of Micromechanics and Microengineering 2008; 18(7): 075001.

30. Boden SA, Bagnal DM. Nanostructured biomimetic moth-eye arrays in silicon by nanoimprint lithography. Proceedings of SPIE 2009; 7401: 74010J.

31. Cheung CL, Nikolic RJ, Reinhardt CE, Wang TF. Fabrication of nanopillars by nanosphere lithography. Nanotechnology 2006; 17(5): 1339-1343.

32. Sun $\mathrm{CH}$, Jiang P, Jiang B. Broadband moth-eye antireflection coatings on silicon. Applied Physics Letters 2008; 92(6): 061112. 